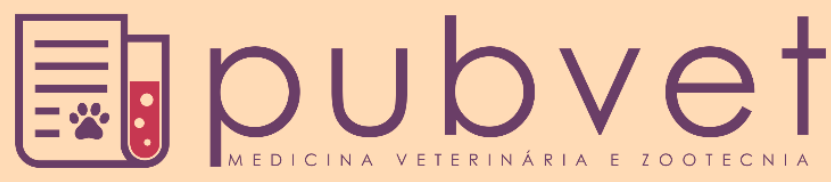

https://doi.org/10.31533/pubvet.v15n06a841.1-7

\title{
Diagnóstico citológico de esporotricose felina na região da Zona da Mata Mineira: Relato de caso
}

\author{
Natalia Amaral de Oliveira ${ }^{0}$, Mônica Maria Altomare de Paula ${ }^{10}$, Cinthya Brillante Cardinot $^{20}$, \\ Thamiris Vilela Pereira Rocha ${ }^{3} \bullet$, Leonardo Lara e Lanna ${ }^{4} \bullet$, Carina Franciscato ${ }^{4 *} \bullet$ (D) \\ ${ }^{1}$ Estudante de graduação em Medicina Veterinária, Universidade Federal de Juiz de Fora (UFJF), Juiz de Fora, Minas Gerais, Brasil \\ ${ }^{2}$ Médica Veterinária, Universidade Federal de Juiz de Fora (UFJF), Juiz de Fora, Minas Gerais, Brasil \\ ${ }^{3}$ Técnica do Laboratório de Patologia Clínica Veterinária, Universidade Federal de Juiz de Fora (UFJF), Juiz de Fora, Minas Gerais, Brasil \\ ${ }^{4}$ Docente do Departamento de Medicina Veterinária, Universidade Federal de Juiz de Fora (UFJF), Juiz de Fora, Minas Gerais, Brasil \\ *Autora para correspondência, E-mail: carinafranciscato@yahoo.com.br
}

Resumo. Apesar de inúmeras suspeitas clínicas, existe uma escassez de dados sobre a ocorrência de esporotricose na região da Zona da Mata Mineira. Assim, este trabalho pretende relatar o primeiro caso de Sporothrix sp. identificado no Laboratório Clínico Veterinário da Universidade Federal de Juiz de Fora (UFJF). Foi atendido na Clínica Veterinária de Ensino da referida instituição, um felino com lesões ulceradas, edemaciadas, hiperêmicas, alopécicas e com focos de necrose em região lateral do tarso e na região interdigital do membro pélvico direito. O exame citológico revelou a presença de inúmeras estruturas leveduriformes fagocitadas e extracelulares, compatíveis com Sporothrix sp. Portanto, o animal passou a ser tratado conforme a prescrição de itraconazol $50 \mathrm{mg}$ SID por 60 dias e uso de terbinafina spray tópica no local da lesão, duas vezes ao dia. Após cerca de dois meses, observou-se que o animal apresentava melhora nas lesões identificadas anteriormente. Porém, o mesmo passou a apresentar uma nova lesão em região frontal da cabeça, com a presença de alterações respiratórias. Então prescreveu-se a associação do iodeto de potássio ao itraconazol, além da utilização de medicação para os sinais respiratórios. No entanto, não houve resposta ao tratamento e ocorreu o óbito do animal. Sabe-se que, a ocorrência de sinais respiratórios está relacionada à refratariedade ao tratamento e morte do animal. Apesar disso, o diagnóstico de esporotricose é fundamental, principalmente utilizando-se o exame citológico, com uma identificação rápida e específica do agente, reduzindo a ocorrência da zoonose. Além disso, destaca-se a importância epidemiológica de descrever o primeiro diagnóstico citológico de Sporothrix sp. realizado no Laboratório de Patologia Clínica Veterinária da UFJF.

Palavras chave: Citologia, felino, Sporothrix sp, zoonose

\section{Cytological diagnosis of feline sporotrichosis in the Forest Zone of Minas Gerais State: Case report}

Abstract. Despite countless clinical suspicions, there is lack of data about sporotrichosis incidence in Zona da Mata Mineira region. Thus, the aim of the current study is to report the first Sporothrix sp. case identified at the Veterinary Clinical Laboratory of Federal University of Juiz de Fora (UFJF). A feline presenting ulcerated, edematous, hyperemic and alopecic lesions, as well as necrotic foci in the lateral region of the tarsus and in the interdigital region of the right pelvic limb was attended at the Veterinary Teaching Clinic of the aforementioned institution. Cytological examination has shown several phagocytized and extracellular yeast structures compatible to Sporothrix sp. Therefore, the animal was treated with $50 \mathrm{mg}$ itraconazole SID for 60 days and with topical terbinafine spray 
application at the lesion site, twice a day. After approximately two months, the animal has shown improvements in the previously identified lesions. However, it presented a new lesion in the frontal region of the head, as well as respiratory changes. Consequently, potassium iodide, in association with itraconazole, was prescribed for the lesion, and medication was prescribed for the respiratory symptoms. Nevertheless, the animal did not respond to the treatment and died. It is known that the incidence of respiratory symptoms is associated with refractoriness to treatment and death. However, it is essential diagnosing sporotrichosis, mainly through cytological examination, to enable quick and specific causative agent identification in order to reduce the incidence of zoonosis. In addition, the current study has emphasized the epidemiological importance of describing the first cytological diagnosis of Sporothrix sp. carried out at UFJF Veterinary Clinical Pathology Laboratory.

Keywords: Cytology, feline, Sporothrix sp., zoonosis

\section{Diagnóstico citológico de esporotricosis felina en la región de la Zona de la Mata Mineira: Relato de caso}

Resumen. A pesar de las numerosas sospechas clínicas, existe una escasez de datos sobre la aparición de esporotricosis en la región de la Zona de la Mata Mineira. Por consiguiente, este trabajo tiene la intención de informar el primer caso de Sporothrix sp. identificado en el Laboratorio Clínico Veterinario de la Universidad Federal de Juíz de Fora (UFJF). En la referida institución, se atendió un felino con lesiones úlceras, edemaciadas, hiperémicas, alopécicas, y con focos de necrosis en la región lateral del tarso y en la región interdigital del miembro pélvico derecho. El examen citológico reveló la presencia de numerosas estructuras leveduriformes fagocitadas y extracelulares, compatibles con Sporothrix sp. Por lo tanto, el animal se trató de acuerdo con la prescripción de itraconazol $50 \mathrm{mg}$ SID durante 60 días y con terbinafina spray tópico en el lugar de la lesión dos veces al día. Después de dos meses, se observó mejoría en las lesiones previamente identificadas. Sin embargo, comenzó a presentar una nueva lesión en la región frontal de la cabeza, con la presencia de alteraciones respiratorias. Se prescribió la asociación del yoduro de potasio con el itraconazol, además del uso de medicamentos para los signos respiratorios. Sin embargo, no respondió al tratamiento y el animal murió. Se sabe que la aparición de signos respiratorios está relacionada con la refractaridad al tratamiento y la muerte del animal. Aunque, el diagnóstico de la esporotricosis es fundamental, principalmente, mediante el examen citológico, con una identificación rápida y específica del agente, reduciendo la aparición de zoonosis. Además, destaca la importancia epidemiológica de describir el primer diagnóstico citológico de Sporothrix sp. realizado en el Laboratorio de Patología Clínica Veterinaria de la UFJF.

Palabras clave: Citología, felino, Sporothrix sp, zoonosis

\section{Introdução}

A esporotricose é uma enfermidade fúngica, caracterizada como zoonose, pois pode ser transmitida dos animais aos humanos (Araujo et al., 2020; Barros et al., 2010). O agente causador dessa enfermidade é um fungo pertencente ao complexo Sporothrix schenckii (Orofino-Costa et al., 2017), classificado como termodimórfico, que apresenta-se de maneira leveduriforme a $37^{\circ} \mathrm{C}$ e na forma micelial à temperatura ambiente (Mezzari \& Manole., 2012).

Os relatos dessa enfermidade iniciaram-se ainda no século XIX. Nessa época e até meados do século XX, essa enfermidade ocorria como surtos epidêmicos (Larsson, 2011) e também podia ser considerada uma ergodermatose ou uma doença essencialmente ocupacional, porque o ser humano infectava-se ao manipular madeiras, solo ou vegetação onde o fungo estava presente (Megid et al., 2016). Entretanto, a partir da década de 1980 ficou conhecido o potencial zoonótico da esporotricose felina e atualmente estes animais são considerados os principais transmissores da enfermidade aos humanos. Os felinos domésticos apresentam características comportamentais que favorecem a sua contaminação com o 
fungo, como o hábito de afiar suas unhas em árvores e madeiras, que são prováveis fontes do agente para infecção do animal, o qual posteriormente provoca arranhadura nos humanos, fazendo a transmissão do fungo pela inoculação traumática (Orofino-Costa et al., 2017). Assim, a esporotricose é uma doença de caráter endêmico em diversos países do mundo (Carlos et al., 2009), inclusive no Brasil, onde vem ocorrendo um aumento significativo de casos clínicos em humanos, principalmente relacionados à transmissão por gatos domésticos (Orofino-Costa et al., 2017).

A manifestação clínica dessa doença nos felinos normalmente se inicia com um nódulo cutâneo ou subcutâneo ulcerado, drenando exsudato serossanguinolento (Larsson, 2011). Algumas manifestações da esporotricose felina podem ser confundidas com outras doenças que promovem alterações cutâneas, tais como a criptococose, micobacterioses tegumentares atípicas, infecções bacterianas profundas e neoplasias como carcinoma de células escamosas, enfatizando a necessidade do diagnóstico laboratorial (Macêdo-Sales et al., 2018).

O diagnóstico de esporotricose pode basear-se em dados da anamnese como topografia da lesão, evolução, exposição a fontes de infecção, além do exame físico com uma análise mais elaborada das lesões apresentadas. Entretanto, o diagnóstico definitivo deve ser amparado por exames laboratoriais, como o exame citológico. Este pode ser realizado através de um imprinting no local da lesão com pus ou outro material, podendo ser examinado a fresco, por microscopia direta, utilizando-se hidróxido de potássio, ou mesmo em esfregaço corado (Orofino-Costa et al., 2017). As colorações convencionais usadas nesse tipo de exame são Gram (Almeida et al., 2018), ou colorações do tipo Romanowsky (Cagnini et al., 2012), as quais permitem que se evidencie estruturas leveduriformes, arredondadas, ovaloides, em forma de "charuto", com 3-5 $\mu$ m de diâmetro e 5-9 $\mu \mathrm{m}$ de comprimento (Larsson, 2011).

Pesquisas já relataram casos de esporotricose tanto em animais quanto em humanos em vários estados brasileiros, mas a maior ocorrência está no sul e sudeste do país (Orofino-Costa et al., 2017), sendo que a esporotricose felina ocorre de maneira endêmica no estado Rio de Janeiro (Megid et al., 2016). A despeito disso, e apesar das inúmeras suspeitas clínicas, existe uma escassez de dados sobre o diagnóstico desta enfermidade na região da Zona da Mata Mineira.

Assim, este trabalho pretende relatar o primeiro caso de Sporothrix sp. identificado no Laboratório Clínico Veterinário da Universidade Federal de Juiz de Fora (UFJF), destacando a importância da citologia na identificação de microrganismos.

\section{Relato do caso}

Foi atendido na Clínica Veterinária de Ensino da UFJF, um felino, macho, não castrado, sem raça definida, com três anos de idade, pesando 4,5 kg, residente no município de Lima Duarte - MG, com relato de lesões ulceradas no membro pélvico direito, apresentando intenso prurido há cerca de 15 dias. Este animal havia sido previamente atendido por um médico veterinário, que suspeitou de esporotricose. Então estava sendo tratado há sete dias, com itraconazol $50 \mathrm{mg}$ (um comprimido SID), amoxicilina + clavulanato de potássio (um comprimido e meio BID) e carprofeno (um comprimido SID).

Ao exame físico observou-se animal em bom estado geral, porém com lesões ulceradas, edemaciadas, hiperêmicas, alopécicas e com focos de necrose em região lateral do tarso (Figura 1A), e na região interdigital do membro pélvico direito (Figura 1B), com animal bastante incomodado ao manuseio.

Realizou-se coleta de amostra de lesão cutânea por imprinting, que foi encaminhada para exame citológico no Laboratório Clínico Veterinário da UFJF. A amostra foi corada por panótico e apresentou intensa celularidade, caracterizada pelo predomínio de neutrófilos segmentados, alguns degenerados, ocasionais linfócitos, macrófagos e células de descamação. Foi observada presença de inúmeras estruturas leveduriformes fagocitadas e extracelulares, compatíveis com Sporothrix sp. (Figura 2). Assim, o animal passou a ser tratado conforme a prescrição de itraconazol $50 \mathrm{mg}$ SID por 60 dias e uso de terbinafina spray tópica no local da lesão, duas vezes ao dia, com suspensão das demais medicações prescritas anteriormente. Foi recomendado, ainda, o isolamento do gato, para evitar contato com outros animais, e a utilização de luvas de procedimento durante qualquer manuseio do animal ou das lesões. Aproximadamente dez dias após o início deste tratamento, o tutor relatou aparente melhora das lesões. 


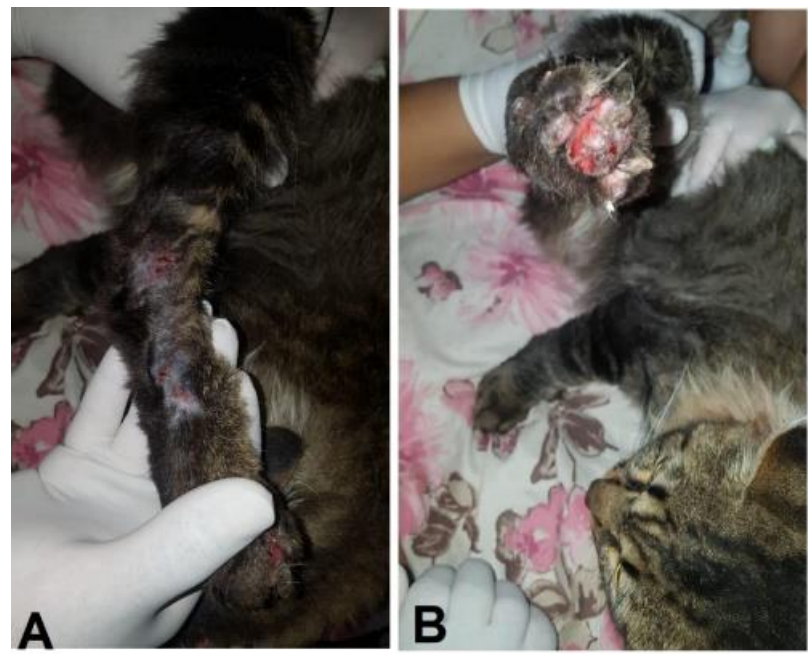

Figura 1. Lesões alopécicas, ulceradas, edemaciadas, hiperêmicas e com focos de necrose em região lateral do tarso (A) e na região interdigital do membro pélvico direito (B) de felino com esporotricose.

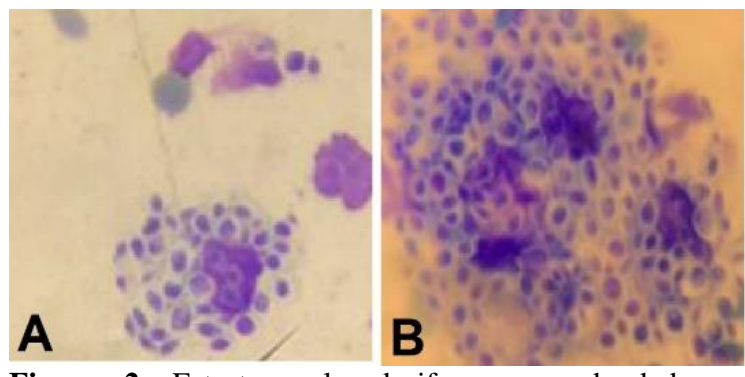

Figura 2. Estruturas leveduriformes arredondadas e ovalóides, fagocitadas (A) e extracelulares (B), compatíveis com Sporothrix sp., observadas em amostras obtidas por imprinting do felino em relato. Visualização em objetiva de imersão (aumento de 1000X).

Foi mantido contato telefônico com os tutores e ao retorno, após cerca de dois meses, observou-se que o animal apresentava melhora nas lesões identificadas anteriormente (Figura 3). Porém, o mesmo passou a apresentar uma nova lesão em região frontal da cabeça (Figura 4), com apresentação de espirros frequentes, ruído respiratório típico de secreção em concha nasal, dispneia e perda de peso. Realizou-se coleta de amostra da nova lesão para exame citológico. Esta análise também revelou a presença estruturas leveduriformes compatíveis com Sporothrix sp. (Figura 5). Então o tratamento para esporotricose foi mantido.
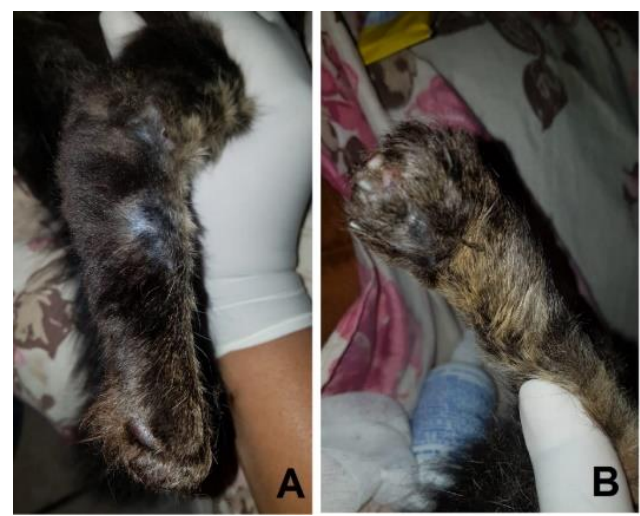

Figura 3. Felino apresentando remissão das lesões cutâneas com aproximadamente dois meses de tratamento.
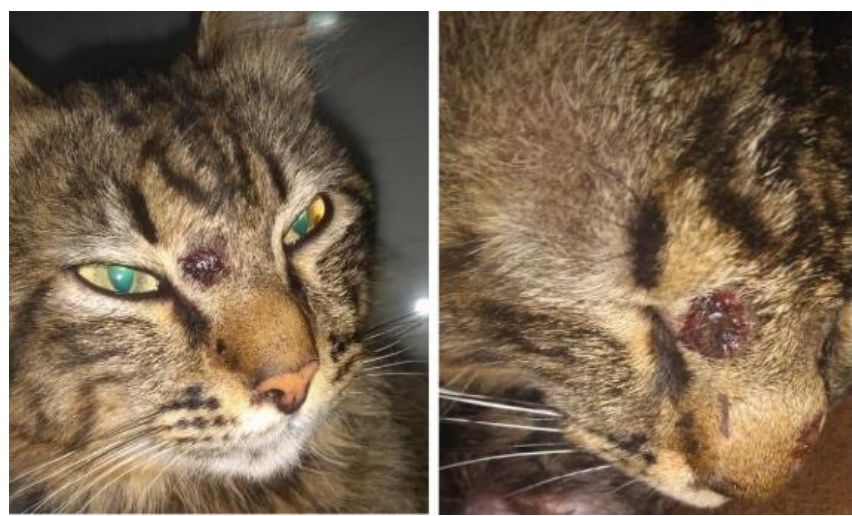

Figura 4. Nova lesão, circunscrita, ulcerada e crostosa, em região frontal da cabeça, apresentada pelo felino mesmo após dois meses de tratamento.

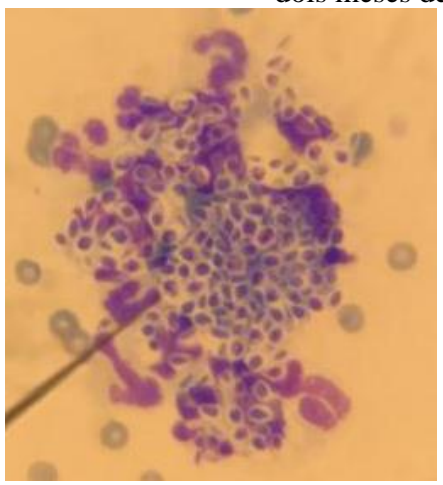

Figura 5. Estruturas leveduriformes compatíveis com Sporothrix sp., observadas em amostras coletadas da nova lesão apresentada pelo felino (região frontal da cabeça). Visualização em objetiva de imersão (aumento de 1000X). 
Aproximadamente quatro dias após essa consulta, o tutor fez contato com o médico veterinário, relatando piora no quadro de dificuldade respiratória do animal. Então foi prescrito o uso de meloxicam $0,5 \mathrm{mg}$ (um comprimido SID por três a cinco dias). Após mais quatro dias o tutor relatou que não houve melhora na respiração e o animal passou a apresentar secreção nasal mucopurulenta. Dessa forma, prescreveu-se prednisona $5 \mathrm{mg}$ (um comprimido BID por cinco dias) para alívio do quadro respiratório, juntamente com a vaporização do ambiente. Em dois dias foi relatada uma discreta melhora na respiração e na secreção. Entretanto, notou-se um aumento de volume nasal.

Quase duas semanas após este episódio, embora tenha apresentado melhora no padrão respiratório e diminuído a secreção nasal, o felino apresentava espirros, diminuição de apetite e emagrecimento. Assim aumentou-se a dosagem do antifúngico e o felino passou a ser tratado com itraconazol $100 \mathrm{mg}$ (uma cápsula SID), associado ao iodeto de potássio $50 \mathrm{mg}$ (uma cápsula SID) até novas recomendações. Também foi prescrito novamente a Prednisona $5 \mathrm{mg}$ (um comprimido BID por cinco dias).

Três semanas após esta prescrição, relatou-se leve melhora, inclusive das lesões da região frontal da cabeça. No entanto, o animal havia apresentado episódio de espirro com sangue. Vinte dias após esta ocasião, foram observadas novas lesões nos membros pélvicos e piora da lesão na face, além de sinais de congestionamento nasal e emagrecimento, sendo que quatro dias após este último relato, ocorreu o óbito do animal.

\section{Discussão}

O animal do presente relato apresentou lesões ulceradas, edemaciadas, hiperêmicas, alopécicas e com focos de necrose em região interdigital, podendo ser confundidas até mesmo com infecções bacterianas, que é um dos diagnósticos diferenciais da esporotricose (Macêdo-Sales et al., 2018). Esta situação destaca a importância dos exames laboratoriais para o diagnóstico definitivo da esporotricose, que neste caso foi a análise citológica. Este tipo de exame é altamente recomendado para o diagnóstico de esporotricose em felinos, por ser simples, prático, de baixo custo, que apresenta resultado rápido e uma eficiência de aproximadamente $80 \%$ na realização do mesmo, segundo demonstrado por alguns autores (Silva et al., 2015), contrastando com o diagnóstico micológico por cultura fúngica, que é um exame mais oneroso e muito mais demorado, pois o crescimento deste fungo geralmente não ocorre antes de cinco a sete dias (Mezzari \& Manole., 2012). Essa morosidade do exame micológico causa uma demora no início do tratamento, o que pode levar ao agravamento das lesões no animal e maior risco de transmissão para outros animais e para o ser humano.

O referido exame foi feito através de um imprinting no local da lesão, e foi examinado em esfregaço corado, como recomendado por Macêdo-Sales et al. (2018). A coloração usada foi o panóptico, uma alternativa disponível na maioria das clínicas veterinárias, que permitiu evidenciar um grande número de formas leveduriformes, arredondadas e ovaloides, conforme descrito por Larsson (2011), sendo que estas estruturas foram observadas dentro e fora de macrófagos, como observado também por Cagnini et al. (2012). Ressalta-se que é comum a ocorrência da fagocitose do Sporothrix como um dos mecanismos da defesa imunológica do hospedeiro, na tentativa de debelar o agente infeccioso (Orofino-Costa et al., 2017).

Além das estruturas fúngicas encontradas, evidenciou-se também a presença de grande quantidade de leucócitos nas lâminas do imprinting realizado no animal, observando-se a predominância de neutrófilos segmentados, sendo alguns íntegros e outros degenerados, além da presença de macrófagos e ocasionais linfócitos. Estas células caracterizam um processo inflamatório, como já descrito por outros autores, em relatos de esporotricose em felinos (Cagnini et al., 2012).

Os felinos, em geral, respondem bem ao tratamento com antifúngicos como cetoconazol, itraconazol e iodetos orgânicos (iodeto de potássio e iodeto de sódio). Destes, o itraconazol tornou-se o medicamento de escolha, devido a sua efetividade e segurança quando comparado aos demais agentes antifúngicos (Gremião et al., 2015) e seu tempo de uso deve ser definido com base na cicatrização das lesões do animal, bem como na demonstração de exames citológicos negativos, sendo essencial o acompanhamento.

No caso relatado, o animal apresentou boa evolução nas lesões interdigitais com o uso do itraconazol sistêmico e terbinafina tópica, que também é um antifúngico que apresenta sucesso no tratamento de esporotricose (Heidrich et al., 2011). O itraconazol e a terbinafina possuem mecanismos de ação distintos e apresentam sinergismo quando associados. No entanto, mesmo ainda recebendo tratamento, este animal 
apresentou recidiva e surgimento de novas lesões cutâneas. Este fato assemelha-se a estudos que já descreveram a recorrência dos sinais clínicos, demonstrando a reativação das lesões (Gremião et al., 2015).

O prognóstico da esporotricose depende do número, extensão e localização das lesões no animal (Greene et al., 1993). Micoses localizadas na região nasal dos felinos são de difícil cura e como alguns animais são refratários ao tratamento apenas com itraconazol, outros medicamentos podem ser utilizados em conjunto, assim como o iodeto de potássio, sendo esta associação indicada para casos graves e refratários (Nelson \& Couto, 2015). Portanto, em razão dos sinais de infecção do trato respiratório superior apresentados pelo felino em relato, optou-se por associar itraconazol e iodeto de potássio. Mesmo assim, o animal não apresentou melhora e acabou indo a óbito. Sabe-se que sinais extra cutâneos da esporotricose podem ser observados nos felinos (Megid et al., 2016), como foi o caso deste animal, que apresentava dispneia, espirros e secreção nasal, e a ocorrência de sinais respiratórios está relacionada à refratariedade ao tratamento e morte do animal (Pereira et al., 2010).

Atualmente a esporotricose tornou-se um grave problema de saúde pública devido ao aumento de casos de transmissão da enfermidade dos felinos para os seres humanos (Megid et al., 2016). Assim, esse relato de caso, mostra a importância da realização de exames diagnósticos em animais suspeitos, além de estudos epidemiológicos na Zona da Mata Mineira, para que se possa registrar a existência da doença na região, o que poderá contribuir para o controle dessa zoonose.

\section{Conclusão}

Devido à importância para saúde pública, o diagnóstico de esporotricose é fundamental. Dessa forma, a identificação do agente deve ser rápida e específica, evitando complicações para os animais e reduzindo a chance de transmissão ao homem, sendo o exame citológico uma opção diagnóstica mais prática e acessível. Além disso, destaca-se a importância epidemiológica de descrever o primeiro diagnóstico citológico de Sporothrix sp. realizado no Laboratório de Patologia Clínica Veterinária da Universidade Federal de Juiz de Fora.

\section{Referências}

Almeida, A. J., Reis, N. F., Lourenço, C. S., Costa, N. Q., Bernardino, M. L. A., \& Vieira-da-Motta, O. (2018). Esporotricose em felinos domésticos (Felis catus domesticus) em Campos dos Goytacazes, RJ. Pesquisa Veterinária Brasileira, 38(7), 1438-1443. https://doi.org/10.1590/1678-5150-PVB-5559

Araujo, A. K. L., Gondim, A. L. de C. L., \& Araujo, I. E. A. (2020). Esporotricose felina e humanarelato de um caso zoonótico. Revista Brasileira de Higiene e Sanidade Animal, 14(2), 237-247. https://doi.org/10.5935/1981-2965.20200021.

Barros, M. B. L., Schubach, T. P., Coll, J. O., Gremião, I. D., Wanke, B., \& Schubach, A. (2010). Esporotricose: a evolução e os desafios de uma epidemia. Revista Panamericana de Salud Publica, 27(6), 455-460.

Cagnini, D. Q., Rodrigues, M. M. P., Palumbo, M. I. P., Heckler, M. C. T., Peixoto, A. S., Amorim, R. L., \& Machado, L. H. A. (2012). Cytologic diagnosis and treatment of feline sporotrichosis: case report. Veterinária e Zootecnia, 19(2), 186-191.

Carlos, I. Z., Sassá, M. F., Sgarbi, D. B. G., Placeres, M. C. P., \& Maia, D. C. G. (2009). Current research on the immune response to experimental sporotrichosis. Mycopathologia, 168(1), 1-10. https://doi.org/10.1007/s11046-009-9190-z.

Greene, C. E., Samperio, J. O., \& Gómez, J. P. (1993). Enfermedades infecciosas: Perros y gatos. Editora Interamericana.

Gremião, I. D. F., Menezes, R. C., Schubach, T. M. P., Figueiredo, A. B. F., Cavalcanti, M. C. H., \& Pereira, S. A. (2015). Feline sporotrichosis: epidemiological and clinical aspects. Medical Mycology, 53(1), 15-21. https://doi.org/10.1093/mmy/myu061.

Heidrich, D., Stopiglia, C. D. O., Senter, L., Vetoratto, G., Valente, P., \& Scroferneker, M. L. (2011). Sucesso terapêutico da terbinafina em um caso de esporotricose. Anais Brasileiros de Dermatologia, 86(4), 182-185. https://doi.org/Heidrich, D., Stopiglia, C. D. O., Senter, L., Vetoratto, G., Valente, P. 
Larsson, C. E. (2011). Esporotricose. Brazilian Journal of Veterinary Research and Animal Science, 48(3), 250-259.

Macêdo-Sales, P. A., Souto, S. R. L. S., Destefani, C. A., Lucena, R. P., Rocha, E. M. S., \& Baptista, A. R. S. (2018). Diagnóstico laboratorial da esporotricose felina em amostras coletadas no estado do Rio de Janeiro, Brasil: limitações da citopatologia por imprint. Revista Pan-Amazônica de Saúde, 9(2), 13-19. https://doi.org/10.5123/S2176-62232018000200002.

Megid, J., Ribeiro, M. G., \& Paes, A. C. (2016). Doenças infecciosas em animais de produção e de companhia. Roca.

Mezzari, A., \& Manole., F. A. (2012). Micologia no laboratório clínico. Manole Ltda.

Nelson, R. W., \& Couto, C. G. (2015). Medicina interna de pequenos animais (Issue 1). Elsevier Editora.

Orofino-Costa, R., Macedo, P. M. de, Rodrigues, A. M., \& Bernardes-Engemann, A. R. (2017). Sporotrichosis: an update on epidemiology, etiopathogenesis, laboratory and clinical therapeutics. Anais Brasileiros de Dermatologia, 92(5), 606-620. https://doi.org/10.1590/abd1806-4841-2017279.

Pereira, S. A., Passos, S. R. L., Silva, J. N., Gremião, I. D. F., Figueiredo, F. B., Teixeira, J. L., Monteiro, P. C. F., \& Schubach, T. M. P. (2010). Response to azolic antifungal agents for treating feline sporotrichosis. The Veterinary Record, 166(10), 290-294.

Silva, J. N., Passos, S. R. L., Menezes, R. C., Gremião, I. D. F., Schubach, T. M. P., Oliveira, J. C., Figeiredo, A. B. F., \& Pereira, S. A. (2015). Diagnostic accuracy assessment of cytopathological examination of feline sporotrichosis. Medical Mycology, 53(8), 880-884. https://doi.org/10.1093/mmy/myv038.

Histórico do artigo:

Recebido: 4 de dezembro de 2020

Aprovado: 3 de fevereiro de 2021.
Licenciamento: Este artigo é publicado na modalidade Acesso Aberto sob a licença Creative Commons Atribuição 4.0 (CC-BY 4.0), a qual permite uso irrestrito, distribuição, reprodução em qualquer meio, desde que o autor e a fonte sejam devidamente creditados. 